\title{
Increased Brain Activation in Motor Cortex After Acupuncture Treatment for Motor Recovery in Chronic Stroke Patients
}

\author{
Anson C.M. Chau, Raymond T.F. Cheung" ${ }^{*}$, Xianyong Jiang, Paul K.M. Au-Yeung and \\ Leonard S.W. Li \\ Department of Medicine (Neurology), Li Ka Shing Faculty of Medicine, The University of Hong Kong, Queen Mary \\ Hospital, Pokfulam, Hong Kong
}

\begin{abstract}
Acupuncture has historically been recommended as adjunctive treatment to patients with stroke, but its effectiveness has yet to be proven. This case series aims to report the results of using functional magnetic resonance imaging (fMRI), together with motor function evaluations, to assess the effect of acupuncture on stable patients after stroke. Eleven chronic stroke patients underwent fMRI and motor function evaluations of their impaired upper limb before and after an 8-week period of acupuncture on two acupoints (Hegu LI-4 and Quchi LI-11), without undergoing simultaneous rehabilitation. Improvements in motor functions of the impaired upper limb were found after acupuncture treatment. Changes in cortical activities were closely related to the finding of improved motor functions. A larger and stronger activation was found in the sensorimotor area of the lesioned hemisphere after receiving the acupuncture intervention. Conjunction analysis demonstrated common regions that were activated during either motor task or acupuncture stimulation. Acupuncture may improve functions of the impaired upper limb in chronic stroke patients via modulating peri-infarct regions that are responsible for plasticity of the motor cortex.
\end{abstract}

\section{INTRODUCTION}

Stroke is a major consequence of cerebrovascular disease. The pathological process of stroke would manifest in one or more blood vessels of the brain to result in ischemia or bleeding in an area of the brain [1]. Stroke is a major cause of death and disability in all societies [2]. Acupuncture has long been used in China for stroke rehabilitation [3], and has been proposed as an adjunct therapy in stroke rehabilitation in Western medicine since $1998[2,4]$. Acupuncture involves stimulation of anatomical locations on the skin by a variety of techniques. The commonest technique used to penetrate the skin is by thin, solid, metallic needles [4]. Acupuncture alters pain threshold via release of neurotransmitters and neuropeptides [5]. Acupuncture also induces changes in regional cerebral blood flow surrounding infarcts [6]. However, to date, no convincing scientific evidence from clinical trials has been published showing acupuncture to be effective as a therapy after stroke in enhancing functional improvement [7-10]. Furthermore, the neural mechanism of acupuncture in stroke patients has not yet been well defined [2].

Functional magnetic resonance imaging (fMRI) studies with healthy subjects have demonstrated cortical activities under acupuncture stimulation. Acupoints, such as Hegu LI4 and Quchi LI-11, that are considered effective for motor impairment of the upper limbs [11], can trigger activities in the sensorimotor cortex when being stimulated [3, 12]. Hegu is located on the dorsum of the hand, lying between the first

*Address correspondence to this author at the Department of Medicine (Neurology), Li Ka Shing Faculty of Medicine, The University of Hong Kong, Queen Mary Hospital, Pokfulam, Hong Kong; Tel: 852-28554049; Fax: 852-28186474; E-mail: rtcheung@hkucc.hku.hk and second metacarpal bones, and approximately in the middle of the second metacarpal bone on the radial side. Quchi is located in the depression at the lateral end of the transverse cubital crease at the elbow [11]. These two acupoints have been widely used in stroke rehabilitation studies $[13,14]$. The present study was conducted to test the hypothesis that promotion of upper limb function after acupuncture would correlate with an increase in cortical activation in the lesioned hemisphere of chronic stroke patients.

\section{MATERIALS AND METHODS}

\section{Subjects}

An established stroke database in a regional hospital was screened for suitable subjects. Patients who had suffered from a single episode of stroke with persistent upper limb motor impairment were recruited. All subjects had undergone intensive rehabilitation during the subacute stage of their strokes. As their strokes had occurred more than nine months ago, they were in the chronic stage [15] upon entry into the present study. Altogether fifteen patients were recruited, and 11 of them finished all acupuncture sessions. Demographic data of these 11 patients are summarized in Table 1. The stroke lesions were located in different sites, and the hand region of the primary sensorimotor cortex was spared. Acupuncture treatment was administered by a registered acupuncturist on an out-patient basis in a Traditional Chinese Medicine Research Centre of a convalescent hospital. Each patient received 24 acupuncture treatments which were given 3 times a week over a period of 2 months. In the beginning of acupuncture, the acupoints Hegu LI-4 and Quchi LI-11 were manually stimulated over the impaired arm. The manual stimulation included twisting and up-thrusting of the acupuncture needles. Adequate 
Table 1. Demographic Data

\begin{tabular}{|c|c|c|c|c|c|c|}
\hline \multirow{2}{*}{ Subject Number } & \multirow{2}{*}{ Gender } & \multirow{2}{*}{ Age } & \multicolumn{3}{|c|}{ Stroke } \\
\cline { 4 - 7 } & & & Chronicity (Months) & Paretic Side & Lesion Sites \\
\hline \hline 1 & F & 45 & 87 & Right & Sensorimotor lacunar \\
\hline 2 & M & 54 & 38 & Right & Internal capsule \\
\hline 3 & M & 58 & 39 & Right & Basal ganglia \\
\hline 4 & M & 54 & 93 & Right & Thalamus and internal capsule & I \\
\hline 5 & M & 43 & 16 & Right & Corona radiata and bilateral thalami & I \\
\hline 6 & M & 72 & 18 & Right & Basal ganglia \\
\hline 7 & M & 66 & 9 & Right & Internal capsule \\
\hline 8 & M & 48 & 25 & Right & Thalama \\
\hline 9 & M & 64 & 22 & Right & Basal ganglia \\
\hline 10 & F & 78 & 12 & Right & Basal ganglia \\
\hline
\end{tabular}

H: hemorrhagic; I: ischemic.

stimulation was indicated by achieving the deqi sensation. In traditional Chinese medicine, deqi refers to a composite of unique sensations and is interpreted as a flow of vital energy, called qi [16]. The stimulation of the acupoints was maintained electrically for 30 minutes by an acupunctosope (Model WQ-6F, Donghua Electronic Instrument Factory, Beijing, China) at a frequency of $2 \mathrm{~Hz}$. During the study period, the patients did not receive any physiotherapy nor occupational therapy.

Tests of impaired upper limb functions and fMRI examinations were conducted at baseline i.e. prior to any study intervention, as well as 9 weeks after completion of the study intervention. Upper limb functions were tested using validated, internationally recognized neurological scales, including Motricity Index [17] (MI), Fugl-Meyer assessment (FMA) [18] and grip force [19]. MRI was conducted using a 3T clinical MRI scanner (Magnetom Trio Tim syngo, Siemens Medical Solutions, Erlangen, Germany) equipped with echo planar imaging (EPI) protocols and a 12-channel head coil receiver.

The $\mathrm{T} 1$-weighted $\left(\mathrm{TR} / \mathrm{TE}=566 / 14 \mathrm{~ms}\right.$; flip angle $=120^{\circ}$; $\mathrm{FOV}=230 \times 230 \mathrm{~mm}$; slice thickness $=3 \mathrm{~mm}$ contiguous) and T2-weighted $\quad\left(\mathrm{TR} / \mathrm{TE}=411 / 92 \mathrm{~ms} ; \quad\right.$ flip angle $=120^{\circ}$; FOV $=230 \times 230 \mathrm{~mm}$; slice thickness $=3 \mathrm{~mm}$ contiguous ) anatomical images, covering the entire cerebral cortex and cerebellum, were obtained in parallel to the intercommissural line for anatomical localization.

Two sets of fMRI data were collected using a T2*weighted EPI sequence $\left(\mathrm{TR} / \mathrm{TE}=3000 / 30 \mathrm{~ms}\right.$; flip angle $=90^{\circ}$; $\mathrm{FOV}=230 \mathrm{X} 230 \mathrm{~mm}$; matrix $=64 X 64$; slice thickness $=3 \mathrm{~mm}$ contiguous; 44 slices; 60 time points for a total of 180s). Each EPI sequence was composed of 4 alternating blocks of 45 s each: rest, activation, rest, and activation. The activation of the first set was achieved by using the impaired arm to perform a hand grip task. The activation of the second set was achieved by stimulation of acupoints LI-4 and LI-11.
The study protocol was approved by the Institutional Review Board of the University of Hong Kong/ Hospital Authority Hong Kong West Cluster. Informed consent was obtained from all subjects.

\section{Data Analysis}

\section{A. Motor Function Evaluations}

Data on motor function assessments before and after acupuncture treatment were compared by paired t-test using the Statistical Package for Social Science software, version 15.0 for Windows (SPSS Inc., Chicago, IL). Data were given in mean \pm SD. A p-value less than 0.05 was taken to infer statistical significance.

\section{B. Functional Magnetic Resonance Imaging Data}

fMRI data were analyzed using SPM2 (Wellcome Department of Cognitive Neurology, London, UK). Contrast images were first created for individual subjects. Next group activations in response to unilateral hand grip movements or acupuncture stimulation of the impaired hand were detected. Group activation images were thresholded at 50 voxels with p-values corrected for multiple comparisons.

Cortical activity during unilateral hand grip task was used to reveal cerebral functional reorganization. It was defined as post-treatment activation exceeded baseline activation using a paired t-test plus an extent threshold of 20 voxels.

To evaluate the influence of acupuncture on motor recovery via cerebral functional reorganization, a conjunction test with family-wise error adjustment plus an extent threshold of 10 voxels was performed to reveal activation sites common to both hand grip task and acupoints stimulation after the course of acupuncture treatment.

Anatomical localization and Brodmann's area (BA) assignment were done using Montreal Neurological Institute (MNI) Space utility [20]. 


\section{RESULTS}

Improved motor functions were documented after acupuncture treatment for 2 months. The MI score was significantly increased from $73 \pm 36.1$ at baseline to $76 \pm 34.1$ (paired t-test, $\mathrm{p}<0.05$ ). The mean FMA score and grip force increased by $1 \%$ and $8 \%$, respectively, but these changes did not reach statistical significance.

Group analysis of baseline fMRI data confirmed activation over the precentral gyrus (BA4) of the lesioned hemisphere during the hand grip task performed by the impaired hand (Fig. 1). After acupuncture treatment, the activation volume for the handgrip task was enlarged to include both precentral and postcentral gyri (BA3, BA4, BA6 and BA40) (Fig. 2). In addition, the activation also appeared to be stronger $(\mathrm{t}$-value $=4.02)$ when compared to the activation at baseline ( $\mathrm{t}$-value $=3.26$ ) (Tables $\mathbf{2}$ and $\mathbf{3}$ ). Peak maxima of activations were different between the baseline and post-treatment fMRI scans in the lesioned hemisphere (Tables $\mathbf{2}$ and $\mathbf{3}$ ).

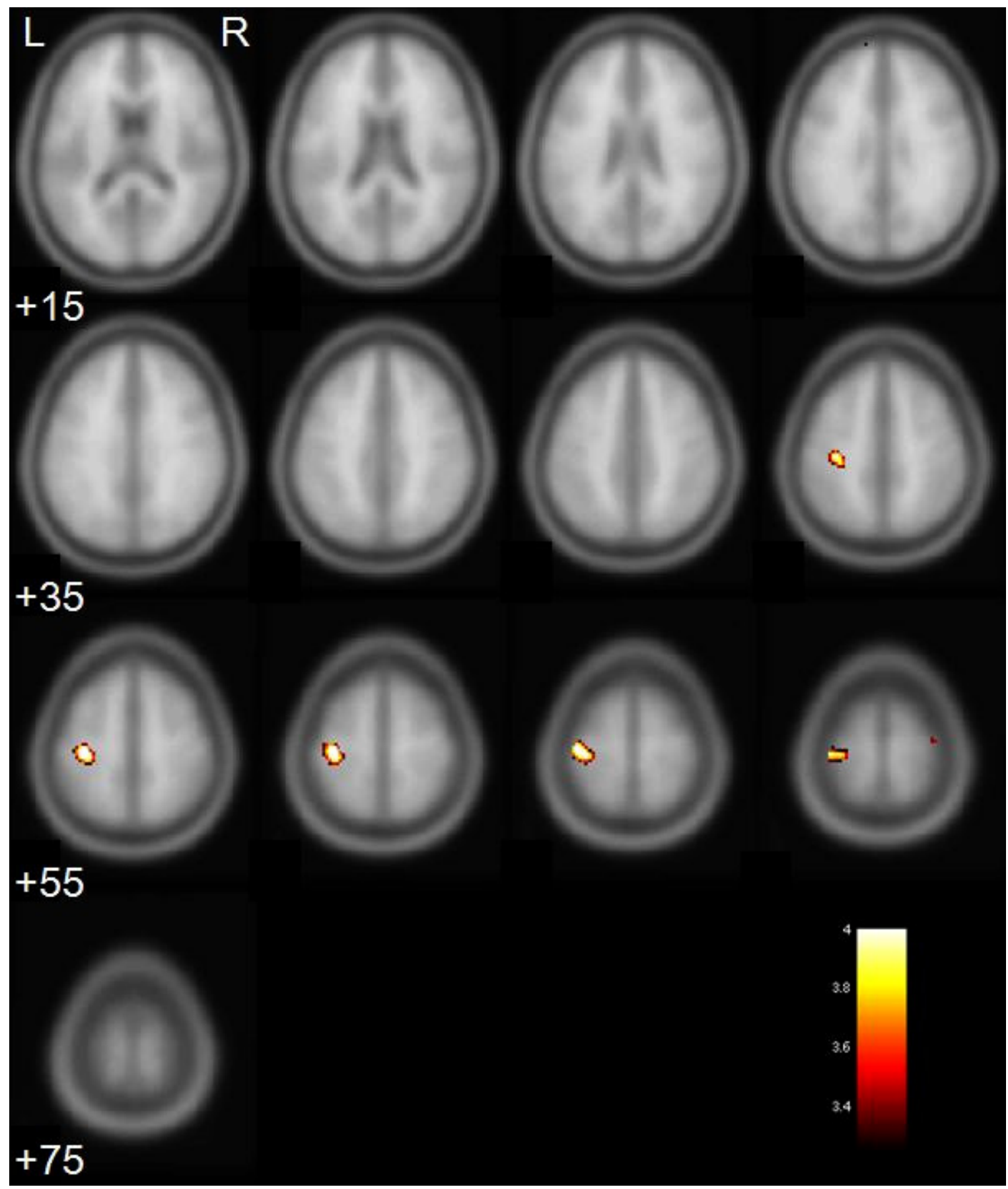

Fig. (1). Activation maps showing significant activation clusters during hand grip task performed by the impaired limb of chronic stroke patients before acupuncture intervention. The numbers below image refer to MNI z-plane coordinates. Each image is $5 \mathrm{~mm}$ apart from each other. The t score scale is shown in the right lower corner. The left hemisphere of the brain is on the left side of the image. 


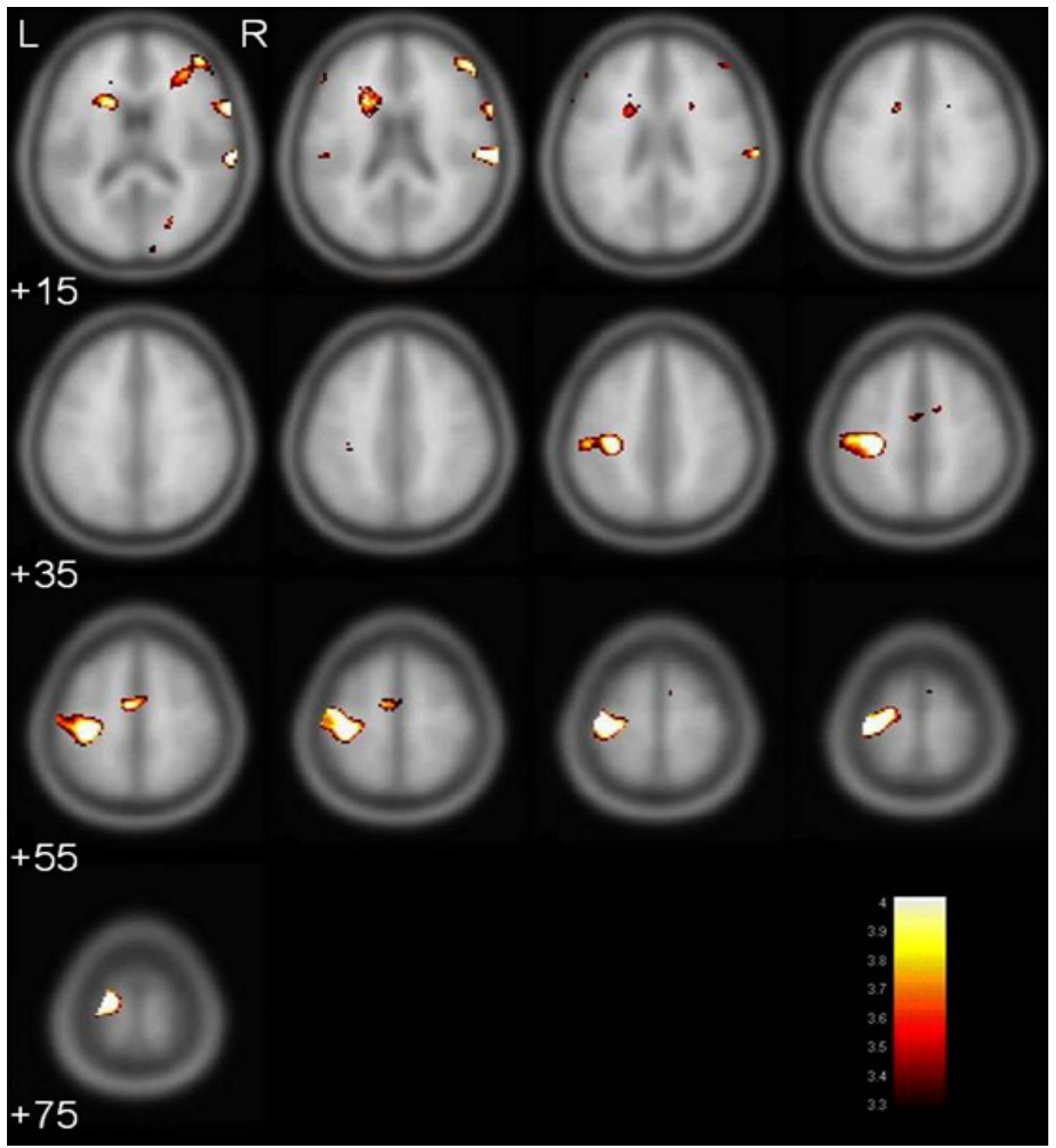

Fig. (2). Activation maps showing significant activation clusters during hand grip task performed by the impaired limb of chronic stroke patients after acupuncture intervention. The numbers below image refer to MNI z-plane coordinates. Each image is $5 \mathrm{~mm}$ apart from each other. The t score scale is shown in the right lower corner. The left hemisphere of the brain is on the left side of the image.

Conjunction analysis revealed that a small region (15 voxels) in the lesioned sensorimotor area $(x=-40, y=-22, z=-$ 68 ; BA4) was activated both during the hand grip task and acupoints stimulation $(\mathrm{p}<0.05)$.

\section{DISCUSSION}

The results of this study support the notion that acupuncture applied to chronic stroke patients with persistent upper limb motor impairment may be beneficial. First, improved motor functions were seen after acupuncture treatment. Second, post-treatment fMRI scan showed increased activation in the lesioned motor cortex during the hand grip task.

Improved functions were detected in chronic stroke patients using all three neurological scales, but only the change in MI score reached statistical significance. A larger sample size is necessary to confirm the potential benefit of acupuncture in motor rehabilitation for chronic stroke patients.
Activation of the motor cortex during the hand grip task was different between baseline and post-treatment in three aspects. First, post-treatment motor cortical activation was larger in volume. Second, post-treatment activation was stronger in magnitude. Third, the peak maxima were differentially localized. A larger and stronger activation is a positive indication of dynamic brain reorganization in order to enhance motor recovery of the impaired upper limb [21]. Displacement of coordinates of the peak maxima may represent cortical rewiring to access the undamaged corticospinal pathways in order to facilitate motor control [22-24]. These observed changes from fMRI scans are in line with the important role of brain plasticity in functional recovery.

A brain imaging study that used single-photon emission computed tomography has shown that acupuncture increases activity in peri-infarct regions within the motor cortex of stroke patients [6]. In the present study, conjunction analysis 
Table 2. Details of Significant Activation Clusters During Hand Grip Task in Chronic Stroke Patients Before Acupuncture Treatment

\begin{tabular}{|c|c|c|c|c|c|c|c|c|}
\hline \multirow{2}{*}{ Clusters } & \multirow{2}{*}{ Hemisphere } & \multicolumn{2}{|c|}{ Coordinates of Global Maxima } & \multirow{2}{*}{$\mathbf{t}$} & \multirow{2}{*}{$\mathbf{p}$} & \multirow{2}{*}{ Regions Involved in the Cluster } & Volumes (mm $\left.\mathbf{m}^{\mathbf{3}}\right)$ \\
\cline { 3 - 6 } & & $\mathbf{x}$ & $\mathbf{y}$ & $\mathbf{z}$ & & & & 54 \\
\hline \hline 1 & Lesioned & -36 & -24 & 56 & 3.26 & 0.001 & Precentral gyrus & 54 \\
\hline
\end{tabular}

Table 3. Details of Significant Activation Clusters During Hand Grip Task in Chronic Stroke Patients After Acupuncture Treatment

\begin{tabular}{|c|c|c|c|c|c|c|c|c|}
\hline \multirow{2}{*}{ Clusters } & \multirow{2}{*}{ Hemisphere } & \multicolumn{2}{|c|}{ Coordinates of Global Maxima } & \multirow{2}{*}{$\mathbf{t}$} & \multirow{2}{*}{$\mathbf{p}$} & \multirow{2}{*}{ Regions Involved in the Cluster } & Volumes (mm $\left.{ }^{3}\right)$ \\
\cline { 3 - 8 } & & $\mathbf{x}$ & $\mathbf{y}$ & $\mathbf{z}$ & & & \\
\hline \hline 1 & Lesioned & -36 & -24 & 70 & 4.02 & 0.000 & Precentral gyrus & 379 \\
\hline & & & & & & & Postcentral gyrus & 114 \\
\hline & & & & & & & Superior frontal gyrus & 73 \\
\hline & Non-lesioned & 66 & -24 & 16 & 3.69 & 0.000 & Puperior temporal gyrus & 7 \\
\hline
\end{tabular}

demonstrated common regions that are activated during either acupuncture stimulation or hand grip task. This is in support of the ability of our selected acupoints to promote motor functional recovery via stimulation of relevant brain areas. Activations induced by our selected acupoints are similar to those seen in a previous study [25], and there is some discrepancies because the previous study stimulated one acupoint instead of stimulation over two acupoints in the present study. The precise mechanism underlying the effect of acupuncture in motor recovery after stroke is unknown.

To the best of our knowledge, this is the first report on longitudinal changes in fMRI signals of chronic stroke patients. Clinical efficacy of acupuncture in stroke rehabilitation in the chronic phase is yet to be confirmed in a controlled clinical trial using a larger sample size. However, the effectiveness of acupuncture is suggested by the present results, as acupuncture stimulation on well recognized motor-implicated acupoints Hegu LI-4 [13, 26-28] and Quchi LI-11 [13, 27-30] was the sole intervention applied during the study period to our clinically stable chronic stroke patients. Such a setting cannot be applied to studies on stroke patients at acute or subacute phase $[13,14,30,31]$ because of ethical considerations. Studies performed to date have been comparisons between acupuncture plus conventional rehabilitation therapy and conventional rehabilitation therapy alone. The effect of acupuncture alone is not easily demonstrable because its beneficial effect would be confounded by the concurrent conventional rehabilitation therapy [9]. However, natural or spontaneous reorganization during the intervention period cannot be ruled out due to the absence of control group in the present study.

Administering treatment intervention to patients who had suffered strokes many months ago is uncommon because rehabilitation is usually recommended as soon as possible after the acute event within 6 months of stroke $[32,33]$ in order to achieve the maximum benefit. A randomized acupuncture study of seven patients whose stroke chronicity was about 4.6 years has revealed a positive correlation between changes in somatosensory-motor function of the impaired upper limb and activation in the ipsilesional motor cortex [34]. However, comparison between the previous study and the present study is difficult because different acupoints and different outcome measures were used. Beneficial responses of acupuncture have also been reported in patients who received acupuncture after 6 years of stroke [35]. However, a randomized controlled study did not find any significant motor improvement in a group of chronic stroke patients who received acupuncture when compared to another group of patients who received blunt needle stimulation on non-existing acupoints [36].

In conclusion, this case series was performed to study the effectiveness of acupuncture for motor recovery in patients with chronic stroke. After the intervention, our results showed improved motor functions in the impaired upper limb. fMRI data revealed a larger and stronger activation in the lesioned sensorimotor motor cortex. Modulations of the motor cortex using acupuncture intervention were also seen. However, absence of a control group of patients is a limitation of our study. Further randomized controlled studies are needed to more thoroughly investigate the efficacy of acupuncture in patients with acute or chronic stroke.

\section{ACKNOWLEDGEMENTS}

We are grateful to Mr. Raymond Lee and his colleagues at Hong Kong Sanatorim \& Hospital MRI centre for their assistance in data collection. We would thank Dr. S.W. Choi for editing the first draft of this manuscript. This study was supported by a General Research Fund from Research Grants Council, HKSAR (HKU7379/04M) and a research grant from Tung Wah Group of Hospitals (20004428). The work forms part of the $\mathrm{PhD}$ thesis submitted to the University of Hong Kong by Anson C.M. Chau. 


\section{REFERENCES}

[1] Special report from the National Institute of Neurological Disorders and Stroke. Classification of cerebrovascular diseases III. Stroke 1990; $21: 637-76$.

[2] Stroke--1989. Recommendations on stroke prevention, diagnosis, and therapy. Report of the WHO Task Force on Stroke and other Cerebrovascular Disorders. Stroke 1989; 20: 1407-31.

[3] Hui KK, Liu J, Makris N, et al. Acupuncture modulates the limbic system and subcortical gray structures of the human brain: evidence from fMRI studies in normal subjects. Hum Brain Mapp 2000; 9: 13-25.

[4] NIH Consensus Conference. Acupuncture. JAMA 1998; 280: 1518-24.

[5] Kim YS, Hong JW, Na BJ, et al. The effect of low vs high frequency electrical acupoint stimulation on motor recovery after ischemic stroke by motor evoked potentials study. Am J Chin Med 2008; 36: 45-54.

[6] Lee JD, Chon JS, Jeong HK, et al. The cerebrovascular response to traditional acupuncture after stroke. Neuroradiology 2003; 45: 7804.

[7] $\mathrm{Wu} \mathrm{HM}$, Tang JL, Lin XP, et al. Acupuncture for stroke rehabilitation. Cochrane Database Syst Rev 2006; 3: CD004131.

[8] Zhang SH, Liu M, Asplund K, Li L. Acupuncture for acute stroke. Cochrane Database Syst Rev 2005; (2): CD003317.

[9] Sze FK, Wong E, Or KK, Lau J, Woo J. Does acupuncture improve motor recovery after stroke? A meta-analysis of randomized controlled trials. Stroke 2002; 33: 2604-19.

[10] Park J, Hopwood V, White AR, Ernst E. Effectiveness of acupuncture for stroke: a systematic review. J Neurol 2001; 248: 558-63.

[11] Deng L, Gan Y, He S, et al. Acupuncture points of the Taiyin and Yangming meridian. In: Cheng X, Eds. Chinese Acupuncture and Moxibustion. Beijing: Foreign Languages Press 1999; 135-63.

[12] Li G, Huang L, Cheung RT, Liu SR, Ma QY, Yang ES. Cortical activations upon stimulation of the sensorimotor-implicated acupoints. Magn Reson Imag 2004; 22: 639-44.

[13] Gosman-Hedstrom G, Claesson L, Klingenstierna U, et al. Effects of acupuncture treatment on daily life activities and quality of life: a controlled, prospective, and randomized study of acute stroke patients. Stroke 1998; 29: 2100-8.

[14] Wong AM, Su TY, Tang FT, Cheng PT, Liaw MY. Clinical trial of electrical acupuncture on hemiplegic stroke patients. Am J Phys Med Rehabil 1999; 78: 117-22.

[15] Sawaki L, Butler AJ, Xiaoyan L, et al. Constraint-induced movement therapy results in increased motor map area in subjects 3 to 9 months after stroke. Neurorehabil Neural Repair 2008; 22: 505-13.

[16] Hui KK, Nixon EE, Vangel MG, et al. Characterization of the "deqi" response in acupuncture. BMC Complement Altern Med 2007; 7: 33.

[17] Wade DT. Measures of motor impairment. In:eds. Measurement in neurological rehabilitation. New York: Oxford Universtiy Press 1992; 154-62.

[18] Gladstone DJ, Danells CJ, Black SE. The fugl-meyer assessment of motor recovery after stroke: a critical review of its measurement properties. Neurorehabil Neural Repair 2002; 16: 232-40.

[19] Sunderland A, Tinson D, Bradley L, Hewer RL. Arm function after stroke. An evaluation of grip strength as a measure of recovery and a prognostic indicator. J Neurol Neurosurg Psychiatry 1989; 52: 1267-72.
[20] Thunberg J, Lyskov E, Korotkov A, et al. Brain processing of tonic muscle pain induced by infusion of hypertonic saline. Eur J Pain 2005; 9: 185-94

[21] Cramer SC, Nelles G, Schaechter JD, Kaplan JD, Finklestein SP, Rosen BR. A functional MRI study of three motor tasks in the evaluation of stroke recovery. Neurorehabil Neural Repair 2001; 15: $1-8$.

[22] Tombari D, Loubinoux I, Pariente J, et al. A longitudinal fMRI study: in recovering and then in clinically stable sub-cortical stroke patients. Neuroimage 2004; 23: 827-39.

[23] Nair DG, Hutchinson S, Fregni F, Alexander M, Pascual-Leone A Schlaug G. Imaging correlates of motor recovery from cerebral infarction and their physiological significance in well-recovered patients. Neuroimage 2007; 34: 253-63.

[24] Pineiro R, Pendlebury S, Johansen-Berg H, Matthews PM. Functional MRI detects posterior shifts in primary sensorimotor cortex activation after stroke: evidence of local adaptive reorganization? Stroke 2001; 32: 1134-9.

[25] Kong J, Ma L, Gollub RL, et al. A pilot study of functional magnetic resonance imaging of the brain during manual and electroacupuncture stimulation of acupuncture point (LI-4 Hegu) in normal subjects reveals differential brain activation between methods. J Altern Complement Med 2002; 8: 411-9.

[26] Hopwood V, Lewith G, Prescott P, Campbell MJ. Evaluating the efficacy of acupuncture in defined aspects of stroke recovery: A randomised, placebo controlled single blind study. J Neurol 2008; 255: 858-66.

[27] Hsieh RL, Wang LY, Lee WC. Additional therapeutic effects of electroacupuncture in conjunction with conventional rehabilitation for patients with first-ever ischaemic stroke. J Rehabil Med 2007; 39: 205-11.

[28] Alexander DN, Cen S, Sullivan KJ, Bhavnani G, Ma X, Azen SP. Effects of acupuncture treatment on poststroke motor recovery and physical function: a pilot study. Neurorehabil Neural Repair 2004; 18: 259-67.

[29] Park J, White AR, James MA, et al. Acupuncture for subacute stroke rehabilitation: a Sham-controlled, subject- and assessorblind, randomized trial. Arch Intern Med 2005; 165: 2026-31.

[30] Sze FK, Wong E, Yi X, Woo J. Does acupuncture have additional value to standard poststroke motor rehabilitation? Stroke 2002; 33: 186-94.

[31] Sallstrom S, Kjendahl A, Osten PE, Stanghelle JH, Borchgrevink $\mathrm{CF}$. Acupuncture in the treatment of stroke patients in the subacute stage: a randomized, controlled study. Complement Ther Med 1996; 4: 193-7.

[32] Small SL, Hlustik P, Noll DC, Genovese C, Solodkin A. Cerebellar hemispheric activation ipsilateral to the paretic hand correlates with functional recovery after stroke. Brain 2002; 125: 1544-57.

[33] Loubinoux I, Dechaumont-Palacin S, Castel-Lacanal E, et al. Prognostic value of FMRI in recovery of hand function in subcortical stroke patients. Cereb Cortex 2007; 17: 2980-7.

[34] Schaechter JD, Connell BD, Stason WB, et al. Correlated change in upper limb function and motor cortex activation after verum and sham acupuncture in patients with chronic stroke. J Altern Complement Med 2007; 13: 527-32.

[35] Naeser MA, Alexander MP, Stiassny-Eder D, Galler V, Hobbs J, Bachman D. Acupuncture in the treatment of paralysis in chronic and acute stroke patients--improvement correlated with specific CT scan lesion sites. Acupunct Electrother Res 1994; 19: 227-49.

[36] Wayne PM, Krebs DE, Macklin EA, et al. Acupuncture for upperextremity rehabilitation in chronic stroke: a randomized shamcontrolled study. Arch Phys Med Rehabil 2005; 86: 2248-55. 